\title{
Associação entre ferramenta de triagem e avaliação nutricional entre pacientes hospitalizados no município de Porto Alegre
}

\author{
Association between screening tool and nutritional assessment among hospitalized patients in \\ the city of Porto Alegre
}

DOI: $10.37111 /$ braspenj.2020352007

Camila Weschenfelder

Luciane Vieira Figueira ${ }^{2}$

Talita Sthephanie Scotta Cabral ${ }^{3}$

Jacqueline Schaurich dos Santos ${ }^{4}$

\section{Unitermos:}

Desnutrição. Avaliação Nutricional. Estado Nutricional.

\section{Keywords:}

Malnutrition. Nutrition Assessment. Nutritional status.

\section{Endereço de correspondência:}

Camila Weschenfelder

Hospital Ernesto Dornelles

Avenida Ipiranga, 1801 - Porto Alegre, RS, Brasil CEP 90160-092

E-mails: camilawesche@gmail.com

\section{Submissão}

9 de maio de 2020

Aceito para publicação

17 de junho de 2020

\section{RESUMO}

Introdução: A desnutrição hospitalar está associada à piora do estado geral do paciente e seu diagnóstico precoce possibilita a redução de complicações relacionadas. O objetivo deste trabalho foi verificar a concordância entre a ferramenta de triagem Short Nutritional Assessment Questionnaire (SNAQ) e a Avaliação Subjetiva Global (ASG). Método: Estudo transversal, realizado em um hospital geral na cidade de Porto Alegre (RS). A SNAQ foi aplicada por nutricionistas e enfermeiros previamente treinados, sendo o paciente considerado em risco nutricional quando o escore foi $\geq 2$. A ASG foi aplicada por nutricionistas em todos os pacientes e considerada padrão-ouro para comparação. O coeficiente Kappa foi utilizado para a avaliação do grau de concordância entre as ferramentas de triagem e avaliação nutricional. Foi considerado valor de Kappa entre 0,2 1-0,60 como baixa concordância, $0,61-0,8$ como concordância moderada e maior que 0,81 , como forte concordância. Resultados: Entre janeiro e março de 2017, foram avaliados 186 pacientes, sendo $115(62 \%)$ mulheres. A média de idade foi de 65,7 $\pm 16,6$ anos e o índice de massa corporal (IMC) médio foi de $26,5 \pm 5,5 \mathrm{~kg} / \mathrm{m}^{2}$. De acordo com a classificação da ASG, $73,7 \%$ da amostra foram considerados bem nutridos, $14 \%$ moderadamente desnutridos e $12,4 \%$ gravemente desnutridos. Foi observada baixa concordância entre o risco nutricional diagnosticado pela enfermagem vs. nutrição por meio da SNAQ (Kappa=0,58) e boa concordância da SNAQ aplicada pela equipe de nutrição com o diagnóstico nutricional da ASG (Kappa = 0,73). A SNAQ apresentou sensibilidade de $85,7 \%$ (IC95\% 73,3 - 92,9) e especificidade de 90,5\% (IC95\% 84,4-94,4); valor preditivo positivo de 76,4\% (IC95\% 65,7 - 84,5) e valor preditivo negativo de 94,7\% (IC95\% 90 - 97,2). Conclusão: Concluímos que a ferramenta de triagem SNAQ, quando conduzida por nutricionistas, pode ser utilizada para detecção precoce da desnutrição hospitalar.

\section{ABSTRACT}

Introduction: Hospital malnutrition is associated with the worsening of the patient's general condition and its early diagnosis allows the reduction of related complications. The aim of this study was to verify the agreement of the Short Nutritional Assessment Questionnaire (SNAQ) screening tool and the Subjective Global Assessment (SGA). Methods: Cross-sectional study, carried out in a general hospital in the city of Porto Alegre (RS). The SNAQ was applied by previously trained nutritionists and nurses, and the patient was considered at nutritional risk when the score was $\geq 2$. SGA was applied by nutritionists in all patients and considered the gold standard for comparison. The Kappa coefficient was used to assess the degree of agreement between the screening and nutritional assessment tools. Kappa values between 0.21-0.60 were considered as low agreement, 0.61-0.8 as moderate agreement and greater than 0.81, as strong agreement. Results: Between January and March 2017, 186 patients were evaluated, of them $115(62 \%)$ were women. The mean age was $65.7 \pm 16.6$ years and the body mass index (BMI) mean was $26.5 \pm 5.5 \mathrm{~kg} /$ $\mathrm{m}^{2}$. According to SGA classification, $73.7 \%$ of the sample was considered to be well nourished, $14 \%$ moderately malnourished and $12.4 \%$ severely malnourished. Low agreement was observed between the nutritional risk diagnosed by nursing vs. nutrition through SNAQ (Kappa =0.58) and good agreement of SNAQ applied by the nutrition team with the nutritional diagnosis of SGA (Kappa $=0.73)$. The SNAQ presented sensitivity of $85.7 \%(95 \% \mathrm{Cl} 73.3-92.9)$ and specificity of $90.5 \%(95 \% \mathrm{Cl} 84.4$ - 94.4); positive predictive value of $76.4 \%(95 \% \mathrm{Cl} 65.7$ - 84.5) and negative predictive value of $94.7 \%$ (95\% CI 90 - 97.2). Conclusion: We conclude that the screening tool SNAQ when conducted by nutritionists can be used for early detection of hospital malnutrition.

1. Nutricionista do Hospital Ernesto Dornelles Especialista e Mestre em Ciências da Saúde: Cardiologia, Porto Alegre, RS, Brasil.

2. Nutricionista pela Universidade Federal de Pelotas, Residente pelo Programa de Residência Integrada em Saúde do Grupo Hospitalar Conceição - RIS/GHC, com especialização em Atenção ao Paciente Crítico, Porto Alegre, RS, Brasil.

3. Nutricionista do Hospital Ernesto Dornelles, Porto Alegre, RS, Brasil.

4. Nutricionista pela Universidade do Vale do Rio dos Sinos, Mestre em Ciências Médicas: Endocrinologia pela Universidade Federal do Rio Grande do Sul. Coordenadora do curso Nutrição do Centro Universitário Ritter dos Reis (UniRitter), Curso de Nutrição, Porto Alegre, RS, Brasil. 


\section{INTRODUÇÃO}

A desnutrição é altamente prevalente em pacientes hospitalizados e, nos países da América Latina, varia entre $40 \%$ e $60 \%$ dos pacientes admitidos nos hospitais'. Pacientes desnutridos e com diminuição da ingestão alimentar na admissão têm maior tempo de internação e piores desfechos clínicos ${ }^{1,2}$. Ademais, desnutrição é preditora dos custos de internação, cujo aumento gira em torno de $19 \%$ a $29 \%{ }^{3}$.

Em 2018, no Brasil, foi lançada a campanha "Diga não à desnutrição" ${ }^{\prime \prime}$, composta por 11 passos para combater a desnutrição hospitalar. Nesta campanha, o primeiro passo engloba a estratificação do risco nutricional e a avaliação nutricional.

Neste sentido, é preciso diferenciar a triagem e a avaliação nutricional. A triagem é um processo rápido e simples. Em ambiente hospitalar, todos os pacientes devem ser triados, a fim de se obter um curso de ação, que também poderá levar à avaliação nutricional ${ }^{4,5}$. Esta define se o paciente é desnutrido ou se possui risco de desnutrição. A avaliação nutricional busca promover o cuidado nutricional por meio da verificação detalhada de variáveis metabólicas, nutricionais e funcionais. Normalmente, é realizada por nutricionista após a triagem, identificando os pacientes que irão se beneficiar da intervenção dietética precoce ${ }^{4,5}$.

Existem várias ferramentas de triagem nutricional validadas e reconhecidas internacionalmente ${ }^{6,7}$; entre elas: Nutritional Risk Screening (NRS), Malnutrition Screening Tool (MST), Mini Nutrition Assessment Short Form (MNASF), Malnutrition Universal Screening Tool (MUST) e Short Nutritional Assessment Questionnaire (SNAQ). A ferramenta $S N A Q^{8}$ foi desenvolvida e validada com o intuito de encontrar um mecanismo de triagem do estado nutricional que fosse válido e de rápida aplicação. A coorte de validação ${ }^{8}$ foi constituída de 297 pacientes, avaliados por enfermeiras na admissão hospitalar, utilizando o escore SNAQ. No entanto, a literatura em relação à aplicabilidade desta ferramenta e, também, a sua associação com desfechos em ambiente hospitalar é escassa ${ }^{9,10}$.

A Avaliação Subjetiva Global (ASG)" é uma ferramenta de avaliação nutricional amplamente utilizada, por ser considerada um método simples, não invasivo, de baixo custo, que pode ser realizado à beira do leito e é capaz de identificar os pacientes desnutridos ${ }^{12}$. A ASG parece apresentar igualdade ou superioridade ao identificar a desnutrição de pacientes clínicos e cirúrgicos quando comparada à antropometria e aos exames laboratoriais ${ }^{13}$.

Conforme a Associação Brasileira de Nutrição (ASBRAN) ${ }^{14}$, a escolha dos instrumentos de triagem e de avaliação nutricional utilizados por cada instituição deve ser definida de acordo com a população (crianças, adultos ou idosos), devendo ocorrer adequado treinamento ao profissional que irá aplicá-los.
Visto que a ferramenta de triagem nutricional adotada deve ser adequada à realidade de cada instituição e, também, predizer corretamente o risco nutricional, o objetivo deste estudo foi avaliar a concordância entre a ferramenta de triagem nutricional $S N A Q$, aplicada pela equipe de enfermagem e pela equipe de nutricionistas, com a ASG, em pacientes internados em um hospital geral, na cidade de Porto Alegre.

\section{MÉTODO}

Trata-se de um estudo transversal, realizado em um hospital geral na cidade de Porto Alegre (RS), em que foram utilizadas informações disponíveis em prontuários médicos e sistemas de informação institucionais. Os dados foram analisados de forma anônima e os resultados foram apresentados de forma agregada, não sendo identificados os participantes de pesquisa. Desta forma, considerando as diretrizes e normas regulamentadoras de pesquisas envolvendo seres humanos descritas na Resolução do Conselho Nacional de Saúde № 466/12 e complementares, não foi necessário aplicar o Termo de Consentimento Livre e Esclarecido (TCLE). $O$ estudo foi cadastrado e aprovado pelo Comitê de Ética em Pesquisa do Hospital Ernesto Dornelles, sob CAAE 65932117.0.3001.5308.

Foram considerados critérios de inclusão pacientes admitidos nas enfermarias e emergência, que foram triados e avaliados nas primeiras 72 horas da admissão hospitalar e com idade $\geq 18$ anos. Foram considerados como critérios de exclusão pacientes que não tiveram condições de responder aos questionários das ferramentas de triagem e avaliação nutricional, ou que não estavam acompanhados de cuidador/familiar que fornecessem as informações para preenchimento do mesmo.

Foram coletados dados de identificação (idade e sexo), de saúde (comorbidades), desfechos clínicos (mortalidade e tempo de internação) e do estado nutricional (triagem e avaliação). Previamente ao início do estudo, a ferramenta SNAQ havia sido incluída na avaliação inicial que é conduzida pela equipe de enfermagem, como uma forma de otimizar o trabalho da equipe de nutrição da instituição. Os enfermeiros haviam sido treinados para aplicação da mesma. No entanto, por se tratar de nova rotina para a equipe de enfermagem, a equipe de nutrição manteve a aplicação da SNAQ. Deste modo, foi possível comparar dados de triagem nutricional coletados pela enfermagem e pela equipe de nutricionistas para os mesmos pacientes. A avaliação nutricional através da ASG foi realizada em todos os pacientes (independente do risco nutricional) e, exclusivamente, por nutricionistas.

A SNAQ ${ }^{8}$ é composta por 3 perguntas: se ocorreu perda de peso involuntária, em caso positivo, se $\geq 6$ kg nos últimos 
seis meses ( 3 pontos) ou se $\geq 3$ kg no último mês ( 2 pontos); se houve redução do apetite ( 1 ponto) e a terceira se usou alimentação líquida ou sonda no mês anterior (1 ponto). Se o paciente tiver uma pontuação um ou zero é considerado sem risco. $\bigcirc$ risco nutricional é considerado quando a pontuação for $\geq 2$ pontos.

A ASG ${ }^{11}$ considera alterações ponderais, na dieta e presença de sintomas, além do exame físico e da demanda metabólica da doença de base para classificação do estado nutricional. Neste estudo, utilizamos a ASG que classifica os pacientes em categorias $A / B / C$ : (A) em bem nutrido, (B) moderadamente desnutrido ou (C) gravemente desnutrido. Para fins de análise, os pacientes com algum grau de desnutrição foram agrupados.

O cálculo do tamanho da amostra foi realizado considerando nível de confiança de $95 \%$, acurácia de $85 \%$, conforme o estudo de validação da $\mathrm{SNAQ}^{8}$, margem de erro de $5 \%$ para mais e para menos, sendo necessários 186 indivíduos.

Para análise estatística, variáveis contínuas foram descritas em média e desvio padrão (DP) ou mediana e intervalo interquartil (IQR) e as categóricas como números absolutos e percentuais. Teste exato de Fisher e Qui-quadrado de Pearson foram utilizados para comparação. Foram apresentados os respectivos intervalos de confiança (IC 95\%) e valores de $\mathrm{p}<0,05$ foram considerados significativos. $\bigcirc$ coeficiente Kappa foi utilizado para a avaliação do grau de concordância entre as ferramentas de triagem e avaliação nutricional. Foi considerado valor de Kappa entre 0,21-0,60 como baixa concordância, 0,61-0,8, como concordância moderada, e maior que 0,81 , como forte concordância.

\section{RESULTADOS}

Entre janeiro e março de 2017, foram avaliados 186 pacientes, sendo 71 (38\%) homens e 115 (62\%) mulheres. A média de idade foi de $65,7 \pm 16,6$ anos e o IMC médio foi de $26,5 \pm 5,5 \mathrm{~kg} / \mathrm{m}^{2}$. Em relação às comorbidades, 91 $(48,9 \%)$ pacientes apresentavam o diagnóstico prévio de hipertensão, $38(20,4 \%)$ de diabetes mellitus, 26 (14\%) de doença arterial coronariana e 13 (7\%) de dislipidemia.

Os pacientes foram triados pelas enfermeiras, em média, em 0,5 $\pm 0,5$ dias, e pela equipe de nutrição, em média, em $1 \pm 0,3$ dias e avaliados pelo nutricionista, em média, em $2 \pm 0,8$ dias. A mediana do tempo de internação foi de 5 dias (IC 95\% 3 - 9). Do total de pacientes incluídos nesta análise, 6 (3,2\%) foram a óbito.

No total, $53(28,5 \%)$ pacientes foram triados pelas enfermeiras e todos os indivíduos foram triados pela equipe de nutrição. A Tabela 1 apresenta a comparação entre o tempo após internação e a aplicação da triagem SNAQ pelas equipes de enfermagem e de nutrição.

\begin{tabular}{lcc}
\hline \multicolumn{3}{l}{ Tabela 1 - Tempo após internação para realizar a triagem SNAQ pelas } \\
enfermeiras e pela equipe de nutrição $[\mathrm{n}(\%)]$
\end{tabular}

SNAQ: Short Nutritional Assessment Questionnaire.

A ASG foi realizada em todos os pacientes e 169 (91,3\%) foram avaliados nas primeiras $72 \mathrm{~h}$ de internação. De acordo com a classificação da ASG, 73,7\% da amostra foram considerados bem nutridos, $14 \%$, moderadamente desnutridos e 12,4\%, gravemente desnutridos.

Foi observada baixa concordância entre o risco nutricional diagnosticado pela enfermagem vs. nutrição por meio da SNAQ (Kappa = 0,58). A triagem nutricional realizada pela equipe de enfermagem subestimou o risco nutricional em $8(14,9 \%)$ pacientes, como, também, superestimou o risco em 2 (3,8\%).

A Tabela 2 demonstra a boa concordância da SNAQ aplicada pela equipe de nutrição com o diagnóstico nutricional da ASG (Kappa =0,73). A ferramenta SNAQ apresentou sensibilidade de $85,7 \%$ (IC95\% 73,3 - 92,9) e especificidade de 90,5\% (IC95\% 84,4 - 94,4); valor preditivo positivo (VPP) de 76,4\% (IC95\% 65,7 - 84,5) e valor preditivo negativo (VPN) de 94,7\% (IC95\% 90 - 97,2).

Tabela 2 - Risco nutricional conforme SNAQ, de acordo com o diagnóstico nutricional pela $A S G[n(\%)]$.

\begin{tabular}{lccc}
\hline & \multicolumn{3}{c}{ ASG } \\
\hline & $\begin{array}{c}\text { Nutridos } \\
\text { (ASG A) }\end{array}$ & $\begin{array}{c}\text { Desnutridos } \\
\text { (ASG B e C) }\end{array}$ & Valor Kappa \\
\hline SNAQ $\leq 1$ & $124(66,7)$ & $7(3,8)$ & \\
SNAQ $\geq 2$ & $13(7)$ & $42(22,6)$ & 0,73 \\
\hline
\end{tabular}

ASG: Avaliação Subjetiva Global; SNAQ: Short Nutritional Assessment Questionnaire.

Observamos uma tendência entre a associação do risco nutricional identificado pela SNAQ aplicada pela equipe de nutrição e óbito $(p=0.06)$. Observamos associação entre desnutrição (ASG B e C) e óbito $(p=0,04)$. O odds ratio $(\mathrm{OR})$ para mortalidade entre os pacientes desnutridos foi de 5,6 (IC95\% 1,05 - 29,5) e entre os pacientes com risco nutricional foi de 4,8 (IC95\% 0,9-25,2). 


\section{DISCUSSÃO}

Neste estudo, demonstramos boa concordância entre a ferramenta de triagem nutricional SNAQ e o diagnóstico nutricional obtido pela ASG entre os pacientes internados em um hospital geral na cidade de Porto Alegre. No entanto, a concordância entre a SNAQ aplicada pela enfermagem quando comparada à equipe de nutrição foi baixa. Acreditamos que este resultado possa ser atribuído à amostra limitada que foi coletada pela equipe de enfermagem, como, também, à menor experiência da equipe de enfermagem com a investigação da história alimentar dos pacientes, já que subestimou o risco nutricional em aproximadamente 15\% da população investigada.

Outro ponto a ser considerado é a viabilidade da aplicação desta ferramenta pela equipe de enfermagem na prática clínica. No nosso estudo, dos 186 pacientes incluídos, apenas $53(28,5 \%)$ foram triados pela equipe de enfermagem. Isso pode ter ocorrido por se tratar de nova rotina, cuja adesão à aplicação precisaria ser melhorada. Entretanto, como a triagem de risco nutricional é essencial para o cuidado nutricional em ambiente hospitalar, é de suma importância que as instituições desenvolvam uma rotina factível neste sentido ${ }^{4}$.

Considerando os dados exclusivamente coletados por nutricionistas, observamos que aproximadamente $30 \%$ dos indivíduos apresentaram risco nutricional. Similarmente, Rabito et al. ${ }^{7}$, entre 752 indivíduos do serviço de emergência de um hospital geral em nível terciário na cidade de Porto Alegre, identificaram risco nutricional em $31,3 \%$ dos indivíduos, de acordo com a ferramenta SNAQ. Além disso, o risco nutricional foi associado ao maior tempo de internação $(p<0,001)$ e à maior mortalidade $(p=0,002)^{7}$.

Diferentemente, um estudo multicêntrico que incluiu 419.086 indivíduos triados pela SNAQ, com média de idade de $62 \pm 18$ anos, observou que 13,7\% destes pacientes estavam em risco nutricional, sendo a maior prevalência deste entre os idosos $(38 \%)^{15}$. Também, os pacientes em risco nutricional apresentavam maior tempo de internação $(+1,43$ dias, 95\% Cl: 1,42, 1,44 d, p < 0,001). Cabe ressaltar que, a média de idade do nosso estudo justifica a semelhança em termos de prevalência com o risco nutricional da população idosa encontrado no estudo citado.

A literatura é escassa quanto à concordância da SNAQ com a ASG. A SNAQ foi correlacionada a variáveis antropométricas e bioquímicas entre 1.000 paquistaneses, que tinham mediana de idade de 51 anos e de IMC 25,9 kg/ $\mathrm{m}^{2}$. Neste estudo, a SNAQ foi negativamente correlacionada ao IMC, hemoglobina e albumina (valor r: $-0.25,-0.242$ e -0.307 , respectivamente; $p<0,001)^{16}$.

método de triagem ideal deve possuir elevada sensibilidade, ou seja, positivo para pacientes que de fato são desnutridos e uma elevada especificidade, negativo para pacientes que não são desnutridos ${ }^{17}$. Comparativamente ao estudo de validação da $\mathrm{SNAQ}^{8}$, encontramos valores superiores com relação à especificidade (83\% vs. 90,5\%), sensibilidade (79\% vs. $85,7 \%)$, VPP (70\% vs. $76,4 \%$ ) e VPN (89\% vs. $94,7 \%$ ). Cabe ressaltar que a validação da SNAQ ocorreu utilizando como padrão-ouro para o diagnóstico de desnutrição a perda de peso $>5 \%$ em um mês ou $>10 \%$ em seis meses; $5-10 \%$ em um a seis meses e/ou IMC <18,5, ao passo que nós utilizamos a ASG como padrão-ouro. A ASG foi escolhida como padrão-ouro para condução desta análise já que é associada ao tempo de internação dos pacientes, complicações infecciosas e não-infecciosas ${ }^{18}$, como, também, parece ser similar à antropometria para o diagnóstico nutricional ${ }^{13}$.

Corroborando com a literatura, observamos maior risco para mortalidade entre pacientes com risco nutricional (OR 4,8) e desnutridos (OR 5,6) 9,19. Leiva-Badosa et al. ${ }^{9}$ utilizaram a SNAQ para avaliar o risco nutricional e identificaram que a mortalidade foi maior entre os pacientes com risco comparativamente aos sem risco nutricional $(50 \%$ vs. $14,2 \%)$. Kang et al. ${ }^{18}$ avaliaram 300 pacientes provenientes de 25 hospitais por meio da ASG e observaram que a taxa de sobrevivência decresceu conforme a piora do estado nutricional (ASG A: $97,9 \%$ vs. ASG B: $90,7 \%$ vs. ASG C: $58,3 \%, p<0,001)$.

Finalmente, é recomendado que a triagem nutricional seja realizada em até 24 h após a admissão hospitalar ${ }^{4}$. Por meio deste estudo, foi identificado que os nutricionistas realizaram 90\% das triagens entre 24 e $48 \mathrm{~h}$ considerando a internação hospitalar. Desta forma, foi necessário desenvolver um método que tornasse possível a triagem em até $24 \mathrm{~h}$ da internação. Por este motivo, o Departamento de Nutrição desta instituição implantou um sistema de classificação em níveis assistenciais, conforme manual de orientação da ASBRAN $^{14}$ para a sistematização do cuidado e, assim, é possível maior dedicação de tempo ao processo de triagens.

O nosso estudo possui limitações: 1. a baixa adesão por parte da equipe de enfermagem pode ter influenciado os resultados em relação à baixa concordância entre a SNAQ aplicada pela enfermagem vs. nutrição; 2. o padrão-ouro utilizado para o diagnóstico de desnutrição foi a ASG, que é dependente do treinamento do avaliador para a sua correta aplicação. No entanto, para minimizar esta interferência, a ASG foi aplicada apenas por 2 nutricionistas treinados; 3 . poucos estudos avaliaram a concordância entre a SNAQ com outras ferramentas, em especial com a ASG, em ambiente hospitalar, tornando a comparação dos nossos resultados com outras populações limitada. 


\section{CONCLUSÃO}

Concluímos que a ferramenta de triagem SNAQ apresentou boa concordância com o diagnóstico nutricional identificado pela ASG. No entanto, observamos baixa concordância entre o resultado da SNAQ obtido pela enfermagem quando comparado à nutrição. Além disso, pacientes em risco nutricional e desnutridos apresentaram maior risco para mortalidade. A SNAQ parece ser um questionário fácil, curto, válido e reprodutível para a detecção precoce da desnutrição hospitalar.

\section{REFERÊNCIAS}

1. Correia MITD, Perman MI, Waitzberg DL. Hospital malnutrition in Latin America: a systematic review. Clin Nutr. 2017;36(4):958-67.

2. Ordoñez AM, Madalozzo Schieferdecker ME, Cestonaro T, Cardoso Neto J,LigockiCamposAC. Nutritional status influences the lenght of stay and clinical outcomes in hospitalized patients in internal medicine wards. Nutr Hosp. 2013;28(4):1313-20.

3. Guerra RS, Sousa AS, Fonseca I, Pichel F, Restivo MT, Ferreira $\mathrm{S}$, et al. Comparative analysis of undernutrition screening and diagnostic tools as predictors of hospitalisation costs. J Hum Nutr Diet. 2016;29(2):165-73.

4. Toledo DO, Piovacari SMF, Horie LM, Matos LBN, Castro MG, Ceniccola GD, et al. Campanha "Diga não à desnutrição": 11 passos importantes para combater a desnutrição hospitalar. BRASPEN J. 2018;33(1):86-100.

5. Kondrup J, Allison SP, Elia M, Vellas B, Plauth M, Educational and Clinical Practice Committee, European Society of Parenteral and Enteral Nutrition (E.S.P.E.N.). ESPEN guidelines for nutrition screening 2002. Clin Nutr. 2003;22(4):415-21.

6. Anthony PS. Nutrition screening tools for hospitalized patients. Nutr Clin Pract. 2008;23(4):373-82.

7. Rabito EI, Marcadenti A, Fink JS, Figueira L, Silva FM. Nutritional Risk Screening 2002, Short Nutritional Assessment Questionnaire, Malnutrition Screening Tool, and Malnutrition Universal Screening Tool are good predictors of nutrition risk in an emergency service. Nutr Clin Pract. 2017;32(4):526-32.
8. Kruizenga HM, Seidell JC, Vet HCW, Wierdsma NJ, van Bokhorst-de van der Schueren MAE. Development and validation of a hospital screening tool for malnutrition: the short nutritional assessment questionnaire (SNAQ). Clin Nutr. 2005;24(1):75-82.

9. Leiva-Badosa E, Badia Tahull M, Virgili Casas N, Elguezabal Sangrador G, Faz Méndez C, Herrero Meseguer I, et al. Hospital malnutrition screening at admission: malnutrition increases mortality and length of stay. Nutr Hosp. 2017;34(4):907-13.

10. Harada K, Ochi K, Taguchi T, Nakamura T, Kanazawa M, Yoshida N, et al. Validity of the Short Nutritional Assessment Questionnaire for Japanese patients with cancer undergoing outpatient chemotherapy. J Med Invest. 2017;64(1.2):117-21.

11. Detsky AS, McLaughlin JR, Baker JP, Johnston N, Whittaker $\mathrm{S}$, Mendelson RA, et al. What is subjective global assessment of nutritional status? JPEN J Parenter Enteral Nutr. 1987;11(1):8-13.

12. Barbosa-Silva MC, Barros AJ. Indications and limitations of the use of subjective global assessment in clinical practice: an update. Curr Opin Clin Nutr Metab Care. 2006;9(3):263-9.

13. Fink JS, Mello PD, Mello ED. Subjective global assessment of nutritional status: a systematic review of the literature. Clin Nutr. 2015;34(5):785-92.

14. Associação Brasileira de Nutrição. Manual orientativo: sistematização do cuidado de nutrição. São Paulo: Associação Brasileira de Nutrição; 2014. [cited 2020 mat 02]. Available from: https:// www.asbran.org.br/storage/arquivos/PRONUTRI-SICNUTVD.pdf

15. Kruizenga H, Van Keeken S, Weijs P, Bastiaanse L, Beijer S, Waal $\mathrm{GH}$, et al. Undernutrition screening survey in 564,063 patients: patients with a positive undernutrition screening score stay in hospital 1.4 d longer. Am J Clin Nutr. 2016;103(4):1026-32.

16. Ali AY, Abdelaziz NE. Short nutritional assessment questionnaire as a malnutrition screening tool for hospitalized patients. Pak J Nutr. 2019;18(7):650-6.

17. Rasmussen HH, Holst M, Kondrup J. Measuring nutritional risk in hospitals. Clin Epidemiol. 2010;2:209-16.

18. CamposFM,MarshallNG.Correlaçãoeacuráciademétodossubjetivos de avaliação do estado nutricional com desfechos clínicos em pacientes cirúrgicos. BRASPEN J. 2019;34(3):258-64.

19. Kang MC, Kim JH, Ryu SW, Moon JY, Park JH, Park JK, et al. Prevalence of malnutrition in hospitalized patients: a multicenter cross-sectional study. J Korean Med Sci. 2018;33(2):e10.

Local de realização do estudo: Hospital Ernesto Dornelles, Porto Alegre, RS, Brasil.

Conflito de interesse: Os autores declaram não haver. 